\section{Le Débat Juridique de la Question de L'Avortement au Canada}

\section{Par Sonia Muhimpundu *}

* Sonia Muhimpundu a obtenu un baccalauréat en Droit et Sociologie à l'Université Laurentienne située à Sudbury en Ontario en 2003. C'est dans ce cadre qu'elle a réalisé son travail Le débat juridique de la question de l'avortement au Canada. Sonia termine actuellement sa première année d'études en Relations internationales à l'Université Laval. Son principal champ de recherche concerne la sécurité humaine.

\begin{abstract}
Abortion is a controversial subject in many societies, and Canada has not been immune to this controversy as demonstrated by the Supreme Court cases of Morgentaler, Borrowoski and Daigle. The outcome of these cases indicated that a woman ought to have the power to establish her own limits regarding the continuation of her pregnancy. These Supreme Court rulings enlighten some important facts. First, like ordinary people, judges have prejudices that guide them in their decision making, even if they do not let it show. Second, the influence of feminism can be strong enough to make judges take a particular stand on their decisions.
\end{abstract}

\section{Introduction}

Le Canada, à l'instar d'autres pays démocratiques, s'est doté d'un système de droit pour éviter l'anarchie à l'intérieur de son territoire et permettre aux individus de vivre dans la paix et l'harmonie. Un système juridique devient, dès lors, essentiel pour faire respecter les droits et libertés des individus. Au Canada, c'est la Charte canadienne des droits et libertés qui jouent se rôle. Dans ce système juridique, le rôle des juges en tant que protecteurs des libertés individuelles est indispensable. Cependant, il arrive que les ceux-ci soient confrontés à des questions sensibles entraînant de grave débat au sein de la société tel que c'est le cas avec l'avortement. L'interruption volontaire d'une grossesse était autrefois punie par la Loi canadienne sur l'avortement, mais cette loi a été invalidée en 1988 pour permettre aux femmes d'être maitresses de leur corps et de recourir à un avortement libre et gratuit. Il sera question ici de ce sujet tant controversé et, pour ce faire, seront examinés les décisions dans les trois affaires: Morgentaler, Borowski et Daigle et suivra, par la suite, une critique de ces trois jugements.

\section{Décisions dans les trois arrêts}

A. Morgentaler

Dans ce procès d'un médecin qui pratiquait des avortements, la Cour suprême du Canada est arrivée à la conclusion, par une majorité de cinq juges contre deux, que la législation sur l'avortement est anticonstitutionnelle. En effet, l'article 251 et 252 du Code criminel ${ }^{1}$ viole l'article 7 de la Charte Canadienne des droits et libertés (article selon lequel chacun a droit à la vie, la liberté et à la sécurité de sa personne). En ce sens, l'article 251 porte atteinte à l'intégrité physique et psychologique d'une femme, en ceci qu'il l'oblige à aller jusqu'au bout de sa grossesse contre son gré. Cette obligation est donc une ingérence au corps de la femme, puisque son avis n'est pas pris en compte. De plus, cet article comprends des procédures complexes par lesquelles une femme doit passer pour obtenir la permission d'avorter, complexité qui constitue en soit une atteinte à la sécurité de la femme enceinte. La seule personne qui s'est légèrement démarquée dans ses motifs est la juge Wilson. Bien qu'elle soit arrivée à la même conclusion que la majorité des juges, elle a fait une analyse différente. Selon elle, la question en litige devrait porter davantage sur l'aspect moral, puisque la femme doit être la seule à prendre une décision sans l'ingérence de l'État. Le fait d'orienter la femme dans sa décision constitue une attaque au droit à la liberté, notamment à la liberté de conscience.

Par ailleurs, lorsqu'on parle d'avortement, on ne peut passer outre l'élément qui le compose et qui en est le cœur, à savoir le foetus, l'enfant non encore né. En effet, dans le cas de l'avortement, le dilemme se pose à savoir si le foetus devrait être considéré ou non comme une personne et si, par conséquent, il devrait jouir des mêmes droits et privilèges que tout citoyen canadien. C'est donc cette question du fottus qui a été traitée par la Cour suprême dans les affaires suivantes.

\section{B. Affaire Borrowski}

\begin{abstract}
${ }^{1}$ Ces articles interdisaient l'avortement et la pratique d'un avortement, sauf si la vie ou la santé de la femme était en danger et cette constatation devrait être faite par le
\end{abstract} comité d'avortement thérapeutique (CAT) qui émettait un certificat d'autorisation. 
Dans cette affaire tranchée en 1989, c'était l'article 251 du Code criminel qui était invoqué par l'appelant. D'après ce dernier, le fœtus est une personne et devrait, par conséquent, être protégé par l'article 7 de la Charte. ${ }^{2}$ La majorité des juges a rejeté cette requête pour cause que cet article avait déjà été jugé inconstitutionnel et que, par conséquent, il n'y avait plus aucune loi à contester. Une autre raison invoquée par la Cour suprême est que, selon le droit anglo-canadien, le fœetus ne faisait pas parti du mot "chacun" de l'article 7. Toutes ces raisons ont donc motivé la Cour suprême à rejeter l'appel.

\section{Affaire Daigle}

Dans cette cause d'origine québécoise, qui date également de 1989, la majorité des juges a emprunté le même raisonnement que dans l'affaire Borowski, à savoir que le fotus n'était pas une personne juridique au sens de la Charte québécoise des droits et libertés et qu'il devait être né vivant afin qu'il puisse bénéficier des droits garantis par cette Charte. De plus, il a été statué dans cette affaire que reconnaître un droit quelconque au fotus reviendrait à écarter les droits accordés aux femmes de disposer de leur corps comme bon leur semble et d'instaurer leurs propres limites quant à la continuation de leur grossesse.

\section{Critique}

\section{A. Emprise des juges}

Dans le système de droit canadien, ce sont les juges de dernière instance (Cour suprême) qui sont appelés à établir le verdict définitif lors d'un procès, c'est-à-dire une décision sans appel que les parties impliquées dans le litige doivent respecter. Toutefois, la Cour suprême ne peut trancher un débat comme bon lui semble, car elle demeure une institution politique qui a des limites. En effet, dans leurs analyses, les juges doivent prendre en considération les conséquences politiques et sociales que leurs décisions entraîneront. Pour cette raison, il devient difficile, voire impossible, de satisfaire les différents groupes d'intérêts qui luttent pour imposer leurs visions au reste de la société. Ainsi, comme l'ont souligné les auteurs Campbell et Pal: «Judges are removed from the daily context of politics.» ${ }^{3}$. Dans le cas de l'avortement, ces groupes d'intérêts qui se sont affrontés, et

\footnotetext{
${ }^{2}$ Selon cet article, «Chacun a droit à la vie, à la liberté et à la sécurité de sa personne; il ne peut être porté atteinte à ce droit qu'en conformité avec les principes de justice fondamentale.»

${ }^{3}$ Robert M., CAMPBELL, Leslie A. PAL. The real words of canadian politics:
} cases in process and policy, Editions Broadview press, 1991, p.47 qui continuent de le faire, sont les mouvements «pro-vie» et «pro-choix». Dans le but de stimuler leurs batailles respectives et d'amorcer des changements politiques, ces mouvements se sont servis de la Cour suprême dans l'espoir que celle-ci fasse une interprétation de la Charte Canadienne des droits et libertés favorable à leur cause respective. De fait, depuis sa promulgation en 1982, la Charte Canadienne des droits et libertés est devenue un outil de droit dont l'interprétation passe par les juges. Cette liberté accordée aux juges a comme effet que ces derniers peuvent interpréter la Charte dépendamment du contexte politique et de l'époque dans lesquels ils se prononcer sur un litige. Toutefois, ce ne sont pas seulement les contextes politiques et temporels qui sont déterminants quant aux décisions des juges. En effet, ces derniers, étant des citoyens comme d'autres, ils prononcent des sentences et font des choix selon leurs vécus, leurs bagages culturels, leurs expériences personnelles, etc. Ils ont eux auss des préjugés, des idées bien arrêtées sur une multitude de sujets, même si cela n'est pas apparent par souci d'impartialité. Cette situation, n'est par ailleurs, pas étonnante, car comme l'ont souligné les auteurs Perrault et Cardinal: «Malgré l'exigence d'objectivité des juges dans le processus d'interprétation des lois [...], ils participent aussi à une communauté » ${ }^{4}$. Par conséquent, la question de l'avortement ne peut pas les laisser indifférents et les motifs de leurs décisions témoignent de la position qu'ils prennent sur ce sujet. En effet, d'une part, il y a les juges qui soutiennent la liberté de choix comme c'est le cas pour Dickson et Lamer qui ont déclaré dans l'affaire Morgentaler que:

«L'article 251 porte clairement atteinte à l'intégrité corporelle, tant physique qu'émotionnelle d'une femme. Forcer une femme, sous la menace d'une sanction criminelle, à mener un fotus à terme sans qu'elle ne satisfasse à des critères sans rapport avec ses propres priorités et aspirations est une ingérence grave à l'égard de son corps.» ${ }^{5}$

Ceci démontre donc le souci d'accorder à la femme le droit de déterminer seule si elle veut ou non garder le bébé qu'elle porte et que sa zone d'autonomie devrait être respectée. Cette approche libérale a, par ailleurs, été soutenue par l'auteure Boivin qui affirme que: «Pour que les

\footnotetext{
${ }^{4}$ Martine, PERRAULT et Linda, CARDINAL « Le droit au choix et le choix au Droit: l'expérience de l'Ontario coalition for Abortion clinics et le jugement Morgentaler », Canadian Journal of Political science, 29, no 2, juin 1996 ${ }^{5}$ R.c.Morgentaler, [1988] 1 R.c.S, p.56-57
} 
femmes soient vraiment égales dans la poursuite de leurs aspirations personnelles, ce sont elles qui doivent avoir le mot final dans la décision d'avorter ou non ${ }^{6} »$.

D'autre part, il y a les juges qui adoptent la position «pro-vie» et qui dénoncent la pratique de l'avortement. Pour eux, cette pratique est considérée comme préjudiciable au fœtus. Or, ce dernier étant sans défense, il devrait être protégé par l'État, parce que comme le soulignent les juges McIntyre et Laforest: «Historiquement, l'existence d'un intérêt public dans la protection des enfants non encore nés a toujours été clairement reconnue et rien ne prouve ni n'indique que le concept de l'avortement à volonté soit généralement accepté dans notre société ${ }^{7} »$. Cette conception se porte garante de la défense des traditions et des valeurs ancestrales. En effet, la position «pro-vie» conçoit une perception conservatrice de la femme par rapport à son rôle fondamental de reproduction. Ce rôle doit donc être préservé en empêchant à la femme d'avorter pour ne pas briser la continuation des générations. Par conséquent, les juges «pro-vie» considèrent qu'il faut garder les lois traditionnelles sans y apporter aucun changement. De ce fait, la lecture de la Charte devrait être restrictive, c'està-dire de ne considérer que le sens premier accorder au droit lorsque la loi a été adopté. En cas de nouveaux problèmes sociaux, il incombera au législateur et non aux juges d'adopter de nouvelles règles, puisque ces derniers ne sont pas élus et que le fait de leur accorder le pouvoir d'invalider une loi reviendrait à remettre en cause la séparation des pouvoirs qui caractérise notre société démocratique. En effet, la société canadienne se caractérise politiquement par le système de démocratie libérale. Dans ce système, le pouvoir législatif est composé de gens démocratiquement élus, contrairement au pouvoir judiciaire qui est constitué de personnes nominées. De ce fait, les juges n'ont aucun droit d'édicter de nouvelles lois ou d'y apporter un quelconque changement, ce rôle étant laissé au législateur.

La question du fœtus soulève, par ailleurs, un autre problème sur le plan sémantique dépendamment de la définition proposée. Pour les uns, il faut : «Considérer la naissance comme le vrai commencement de la vie humaine, car c'est à ce moment que l'enfant est accueilli au sens de la collectivité.» ${ }^{8}$, alors que pour d'autres: «Le fotus est une personne en

${ }^{6}$ France, PARENT. L'avortement: les enjeux actuels, Québec, Éditions GREMF, 1990

${ }^{7}$ R.c.Morgentaler, [1988] 1 R.c.S, p.56-57

8 Anne, COLLINS. L'avortement au Canada, Montréal, Éditions Remue-ménage, 1985 , p. 272 miniature semblable à touts égards au nouveau-né et le droit, par conséquent, ne devrait donc pas faire une distinction entre le bébé et l'enfant non encore né.»9 . Cette diversité d'opinions sur la nature du fœetus explique, du moins en partie, pourquoi les trois affaires ont soulevé tant de remous et pourquoi la décision sur l'avortement a été lente puisque la bataille juridique concernant ce sujet a débuté en 1970 pour se terminer en 1988 .

\section{B. Influence du féminisme}

Il a été évoqué dans les lignes précédentes que les juges sont les seuls individus à pouvoir doter la Charte d'une signification en cas d'ambiguïtés qui ne manquent pas dans la Charte Canadienne. Pour remédier à ces ambivalences, les juges interprètent la Charte de façon téléologique ; ils prennent en considération l'objet d'une loi et se penchent sur l'objectif qu'avait le législateur lorsqu'il l'a adopté. Cette intériorisation octroie aux juges de la Cour suprême du Canada une marge de manœuvre. Ceux-ci peuvent ainsi faire échec aux législateurs lorsque ces derniers ne se conforment pas à la Charte dans l'adoption des règlements, des normes et des lois. Mais, également, si la législation ne prend pas en considération les réalités de la société qu'elle dessert. Ces réalités doivent, en fait, être prises en considération puisqu'elles évoluent d'une génération à l'autre, ce qui oblige la Cour suprême à ajuster continuellement le droit. C'est justement cette adaptation du droit qu'il a fallu réaliser dans l'affaire Morgentaler. Cette décision fut déterminante et marquante dans la progression des conditions des femmes. Il faut aussi dire que ce verdict ne fut pas facile à rendre et la durée du débat de Morgentaler (1970-1988) le témoigne. Le questionnement qui se pose désormais est de savoir ce qui a poussé la Cour suprême du Canada à renverser le verdict des instances inférieures.

Pour répondre à ce questionnement, il faut, tout d'abord, tenir compte du rôle que les femmes ont joué dans ce débat. Les premiers mouvements féministes, qui ont émergé au cours du XIXe siècle, réclamaient le droit pour le contrôle de leur corps. Par conséquent, ils contestaient ouvertement la loi sur l'avortement. Les mouvements féministes avaient également d'autres requêtes concernant le droit de vote et l'accès à l'éducation, etc. Avec le temps, les protestations féministes sont devenues plus puissantes et organisés. Les femmes ont finalement réussi à acquérir des droits comme celui de voter, ce qui leurs a permis de se frayer

${ }^{9}$ COMMISSION DE REFORME DU DROIT DU CANADA, Les crimes contre le fotus, document de travail \# 58, Ottawa, Information Canada, 1989 
un chemin dans la vie publique et d'être représentées dans les décisions politiques. C'est ainsi qu'on a commencé à nommer des femmes juges à la Cour suprême du Canada qui ne pouvait plus ignorer la portée da la force politique que représentait les mouvements féministes. Ainsi, la décision finale prise par la Cour supérieure au sujet de l'avortement:« Reflected the emergence of feminism as a major force in canadian politics. The growth of feminist influence in canadian political, educational and legal elites was a necessary pre-condition for the Morgentaler's decision. » 10

En outre, le droit à l'avortement libre accordé aux femmes s'inscrit dans une perspective progressiste. En effet, les femmes forment un groupe qui a toujours été tenu à l'écart, marginalisé par une société qui se voulait patriarcale. Par conséquent, il a fallu plusieurs batailles pour qu'on reconnaisse enfin les femmes comme étant des personnes égales aux hommes et que la loi devrait les protéger tout autant. Le droit à l'avortement est dès lors devenu une condition nécessaire pour compléter les droits accordés à chaque femme en tant qu'individu. En ce sens, le droit à l'avortement consiste en un bouclier contre les abus de toutes sortes que les femmes peuvent subir à l'égard de leurs corps, puisqu'il leurs accorde la possibilité de décider en toute tranquillité si elles veulent ou non poursuivre leur grossesse.

Dans ce cas, on constate que la révolution des femmes, comme toute révolution d'ailleurs, a conduit à l'élaboration d'une nouvelle norme applicable aux femmes, ce qui leur permet à l'avenir de contrôler leur fécondité. À partir de ce moment, le droit devient un outil de lutte pour les groupes d'une société qui veulent entamer des transformations dans leurs conditions de vie. En ce sens, on peut dire que le droit peut être considéré comme la cristallisation d'un rapport de force à une époque donnée et ce pour entamer des changements sociaux importants; changements qui doivent, par ailleurs, être codifiés afin qu'ils puissent être respectés par la collectivité.

\section{Place du citoyen dans la Charte}

Malgré la décision d'accorder le droit aux femmes de recourir à une interruption de la grossesse libre et gratuite, la Cour a néanmoins accepté d'entendre les causes de Borowski et Daigle qui défendaient les droits du fotus et qui insistaient sur la réinstauration de la constitutionnalité de l'article 251 du Code criminel. La Cour a rejeté ces appels dans la mesure

${ }^{10}$ F.L., MORTON. Morgentaler V. Borowoski: Abortion, the Charter and the Courts, Toronto, Éditions McClelland \& Stewart, 1992, p.296 où elle ne pouvait plus revenir en arrière à propos des droits qu'elle venait d'accorder aux femmes dans l'affaire Morgentaler. Ces pourvois étaient donc purement théoriques et la Cour aurait pu refuser de trancher ces affaires qui ne soulevaient qu'une question hypothétique. D'ailleurs, ces deux affaires prouvent que les juges agissent d'une manière tel qu'ils ne veulent pas heurter un individu ou un groupe donné en refusant de lui tendre l'oreille. Si la Cour suprême avait refusé d'accorder l'audience aux parties, le mouvement «pro-vie» se serait sans douté soulevé, ce qui par ailleurs aurait eu des retombées majeures sur le plan politique.

Une observation similaire peut être tirée de ces deux affaires dans la mesure qu'en acceptant d'écouter Borowski et Daigle, les juges voulaient démontrer également que tout individu a le droit d'être entendu devant la Cour et que, par conséquent, il ne fallait pas privilégier une personne plutôt qu'une autre. En agissant ainsi, la Cour suprême prouve qu'elle n'a aucun parti pris dans les litiges qu'elle doit trancher et qu'elle demeure indépendante et impartiale en toute circonstance. De cette manière, elle conserve la confiance des citoyens qui continuent à la supporter. Cette attitude des juges fondée sur le principe de la primauté du droit fait honneur au libéralisme contenu dans la Charte Canadienne, car celle-ci énonce à l'article 15 que tous les citoyens sont égaux devant la loi et que la clause du contrat social s'applique à toute la collectivité sans exception.

La place du citoyen s'observe dans la Charte Canadienne par la notion de "personne". En effet, dans plusieurs articles de la Charte, notamment ceux qui touchent l'avortement, on retrouve la notion de "personne" pour désigner les citoyens. Par exemple, comme il a été mentionné, l'article 7 stipule que "chacun a droit à la vie, à la liberté et à la sécurité de sa personne" (non en italique à l'origine).C'est cette notion qui est au cœur du litige dans les trois arrêts mentionnés. En effet, les différents groupes d'intérêts se sont basés sur ce mot pour convaincre le juge sur le bien fondé de leurs croyances. Pour les partisans du pro-choix, le mot "personne" signifie que les femmes doivent avoir plus de liberté. Mais également, les obliger d'avorter serait une exigence qui porterait atteint à leur sécurité dans la mesure où l'acte d'avorter peut être considéré comme un geste dangereux pour la vie de la femme enceinte. Pour les défenseurs de la vie, le mot "personne" suppose que le fœtus doit bénéficier des mêmes privilèges que le reste de la société. L'État doit donc renforcer sa protection envers l'enfant non encore-né puisqu'il s'agit d'un être vulnérable. La résultante est que: "The person's case has come to be celebrated both as a 
model of flexible, pragmatic constitutional interpretation, as well as a model of judicial promotion of human rights. ${ }^{11}{ }^{\prime}$

Qui a raison? Qui a tort? Il serait difficile de le dire, car les deux groupes antagonistes ont apporté des arguments solides pour appuyer leurs croyances. Néanmoins, la Cour suprême du Canada a tranché que les droits de la femme devraient l'emporter sur ceux du fotus, ce qui l'a amené à conclure à l'inconstitutionnalité de l'article 251 du code criminel.

\section{Conclusion}

En somme, la série de poursuites intentée contre Morgentaler, ainsi que les procès de Borowski et Daigle nous montre la délicatesse du débat sur l'avortement. En effet, ces causes, faisant appel à des visions divergentes, ont dû recourir aux jugements de la Cour suprême du Canada soit pour dénoncer soit pour appuyer la loi sur l'interruption volontaire d'une grossesse. Cette situation démontre toute la complication d'une prise de décision en ce qui a trait à la vie des citoyens en général et à celle des femmes en particulier. La réalité sociale étant complexe, elle peut difficilement obtenir l'approbation de tous et chacun sur un sujet donné. Mais faut-il, comme l'a préconisé McIntyre dans son jugement dans l'affaire Morgentaler, vivre la réalité d'un individu ou d'un groupe (notamment les femmes dans ce cas-ci) pour le comprendre?

Quoi qu'il en soit, l'avortement, qui au départ est un sujet privé, est devenu une affaire publique depuis cette trilogie. C'est d'ailleurs pour cette raison que les protestataires de la décision finale ont voulu réanimer le débat en adoptant la loi C-43 (pour criminaliser une autre fois la pratique de l'avortement), loi qui a échoué. Enfin, le débat sur l'interruption libre de grossesse jette une lumière sur l'influence et l'emprise que peuvent exercer certains groupes sociaux sur le système judiciaire pour que soient adoptées certaines lois. Le droit constitue donc un havre mobile qui permet à une société de changer, d'évoluer pour que tous les individus d'une même collectivité puissent atteindre une l'égalité.

\section{Bibliographie}

R.c.Morgentaler, [1988] 1 R.C.S

R.c.Borowski, [1989] 1 R.C.S.

Tremblay c. Daigle, [1989] 3 R.C.S

${ }^{11}$ Ibid. p. 137
MINISTERE DE LA JUSTICE, Charte canadienne des droits et libertés,

PARENT, France. L'avortement: les enjeux actuels, Québec, Éditions GREMF, 1990

CAMPBELL, Robert et Leslie, A., PAL. The real words of canadian politics: cases in process and policy, Peterborough, Editions Broadview press, 1994, 338 p.

COLLINS, Anne. L'avortement au Canada, Montréal, Éditions Remue-ménage, 1987, 319 p.

COMMISSION DE REFORME DU DROIT DU CANADA, Les crimes contre le fotus, document de travail \# 58, Ottawa, Information Canada, 1989

MORTON, F. L. Morgentaler v. Borowski: Abortion, the Charter, and the Courts, Toronto, Éditions McClelland \& Stewart, 1992,

PERRAULT, Martine et Linda CARDINAL. « Le droit au choix et le choix au Droit: l'expérience de l'Ontario coalition for Abortion clinics et le jugement Morgentaler », Canadian Journal of Political science, 29, no 2 , juin 1996 